\title{
Intraoperative measurement of acetabular component position using imageless navigation during revision total hip arthroplasty
}

\author{
Xin Yu Mei, MD \\ Ali Etemad-Rezaie, MD \\ Oleg A. Safir, MD \\ Allan E. Gross, MD \\ Paul R. Kuzyk, MD
}

Accepted Oct. 5, 2020

\author{
Correspondence to: \\ X.Y. Mei \\ Department of Orthopaedic Surgery \\ Mount Sinai Hospital \\ 600 University Ave \\ Rm 476A \\ Toronto ON M5G 1X5 \\ xmei@qmed.ca
}

DOI: $10.1503 /$ cjs. 012420

\begin{abstract}
Background: Acetabular component malposition is a major cause of dislocation following total hip arthroplasty (THA). Intellijoint HIP is an imageless navigation tool that has been shown to provide accurate intraoperative measurement of cup position during primary THA without substantially increasing operative time. However, its accuracy in revision THA has not been evaluated. This study therefore aims to assess the accuracy of Intellijoint HIP in measuring cup inclination and anteversion in comparison with computed tomography (CT) during revision THA.
\end{abstract}

Methods: Intellijoint HIP was used to measure the position of the preexisting cup in 53 consecutive patients undergoing revision THA between December 2018 and February 2020. Two authors blinded to the intraoperative navigation measurements also independently measured cup position using preoperative CT according to Murray's radiographic definitions. Pearson correlation coefficients with $95 \%$ confidence intervals (CIs), paired $t$ tests and Bland-Altman plots were used to assess agreement between navigation- and CT-measured cup position. Statistical analysis was performed using GraphPad Prism, with $p$ values less than 0.05 indicating statistical significance.

Results: There was excellent agreement between navigation and CT measurements for both cup inclination $(r=0.89,95 \%$ CI $0.81-0.93)$ and anteversion $(r=0.93,95 \%$ CI $0.88-$ 0.96 ), with the mean absolute difference being $5.2^{\circ}$ (standard deviation [SD] $4.0^{\circ}$ ) for inclination and $4.8^{\circ}\left(\mathrm{SD} 5.4^{\circ}\right)$ for anteversion. The navigation measurement was within $10^{\circ}$ of the radiographic measurement in 47 of $53(88.7 \%)$ cases for inclination and 46 of 53 $(86.8 \%)$ cases for anteversion.

Conclusion: Imageless navigation demonstrated excellent correlation and agreement with CT measurements for both inclination and anteversion over a wide range of acetabular component positions.

Contexte : La malposition de la composante acétabulaire est une cause majeure de luxation après une intervention pour prothèse totale de la hanche (PTH). Intellijoint HIPMD est un outil de navigation sans image qui offre une mesure peropératoire précise de la cupule acétabulaire lors d'une PTH primaire, sans prolonger indument le temps opératoire. Par contre, sa précision lors d'une révision de PTH n'a pas été vérifiée. Cette étude vise donc à évaluer la capacité d'Intellijoint HIP à mesurer précisément l'inclinaison et l'antéversion de la cupule acétabulaire en comparaison avec la tomodensitométrie (TDM) lors d'une révision de PTH.

Méthodes : Intellijoint HIP a été utilisé pour mesurer la position de la cupule préexistante chez 53 patients consécutifs soumis à une révision de leur PTH entre décembre 2018 et février 2020. Deux auteurs non informés des mesures peropératoires obtenues avec l'outil de navigation ont aussi mesuré de façon indépendante la position de la cupule à l'aide d'une TDM préopératoire selon les définitions radiographiques de Murray. Nous avons utilisé les coefficients de corrélation de Pearson avec des intervalles de confiance (IC) à $95 \%$, des tests $t$ appariés et des graphiques Bland-Altman pour évaluer la concordance entre la position acétabulaire mesurée avec l'outil de navigation et par TDM. L'analyse statistique a été effectuée avec GraphPad Prism et les valeurs $p$ inférieures à 0,05 indiquaient une portée statistique.

Résultats : On a observé une excellente concordance entre les mesures obtenues avec l'outil de navigation et par TDM pour l'inclinaison $(r=0,89$, IC à $95 \% 0,81-0,93)$ et l'antéversion de la cupule $(r=0,93$, IC à $95 \% 0,88-0,96)$, l'écart absolu moyen étant de $5,2^{\circ}$ (écart-type [É.-T.] $4,0^{\circ}$ ) pour l'inclinaison et de $4,8^{\circ}$ (É.-T. $5,4^{\circ}$ ) pour l'antéversion. $\mathrm{La}$ mesure au moyen de l'outil de navigation s'est retrouvée à l'intérieur d'une limite de $10^{\circ} \mathrm{par}$ rapport à la mesure radiographique dans 47 cas sur $53(88,7 \%)$ pour l'inclinaison et 46 cas sur $53(86,8 \%)$ pour l'antéversion.

Conclusion : La navigation sans image a donné lieu à une corrélation et une concordance excellentes avec les mesures par TDM pour l'inclinaison comme pour l'antéversion et ce, dans un vaste éventail de positions de la composante acétabulaire. 
D islocation following total hip arthroplasty (THA) is a common complication associated with substantial morbidity and increased health care expenditures. A 2009 study of more than 50000 revision THAs found dislocation to be the most common reason for revision following primary THA, accounting for $22.5 \%$ of all revisions and $33 \%$ of acetabular revisions. ${ }^{1}$ Although the causes of dislocation are often multifactorial, many authors agree that cup malposition is one of the most important modifiable risk factors for dislocation. Although controversy exists regarding its inability to account for pelvic tilt and spinopelvic mobility, ${ }^{2}$ the Lewinnek safe zone of $40^{\circ}$ plus or minus $10^{\circ}$ inclination and $15^{\circ}$ plus or minus $10^{\circ}$ anteversion remains a widely accepted target for cup position in modern clinical practice. ${ }^{3}$

Currently, there is no consensus regarding the optimal method to accurately position the acetabular component within a predetermined safe zone. The most commonly used intraoperative aids are manual mechanical alignment guides that attach onto the cup impactor and allow the surgeon to visually assess cup inclination in reference to the floor, and cup anteversion in reference to the patient's ipsilateral shoulder. However, studies have shown substantial variability in cup position among acetabular components placed using mechanical alignment guides, with a large number of components being outside the Lewinnek safe zone., ${ }^{4,5}$ Computerassisted navigation systems have also been developed to assist in acetabular component positioning. Navigation using computed tomography (CT) has been shown to result in more accurate cup positioning, but it is associated with increased operative time and radiation exposure. ${ }^{6,7}$ In comparison, imageless navigation uses intraoperatively registered bony landmarks and mounted sensors to provide real-time data on cup position without the need for preoperative CT or intraoperative fluoroscopy. Despite these advantages, imageless navigation has not been widely adopted into routine clinical practice. Potential reasons for the slow adoption include the cost of the associated equipment, the paucity of literature validating the accuracy of this approach and the increased operative time; studies have reported that the operative time for cases using imageless navigation is up to 58 minutes longer than that for non-navigated cases. ${ }^{8}$

Intellijoint HIP (Intellijoint Surgical) is an imageless navigation tool that has been shown to provide accurate intraoperative measurement of cup position during primary THA, ${ }^{9}$ while increasing mean operative time by only 2.9 minutes. ${ }^{10}$ However, its accuracy in revision THA has not been evaluated. This study therefore aims to assess the accuracy of Intellijoint HIP in measuring cup inclination and anteversion during revision THA, as determined by the level of agreement between intraoperative navigation measurements and radiographic measurements derived from preoperative CT.

\section{Methods}

Following approval of the study by the Mount Sinai Hospital Research Ethics Board (project 19-0184-C), Intellijoint HIP was used to intraoperatively measure the inclination and anteversion of the preexisting acetabular component in a prospective cohort of 53 consecutive patients who underwent revision THA between December 2018 and February 2020 at Mount Sinai Hospital in Toronto, Ontario. Preoperative CT was performed in all patients as part of the preoperative planning process. All procedures were performed in the lateral decubitus position by a single surgeon using standard positioning instruments and retractors. The position of the preexisting cup was measured before extensive scar tissue débridement and manual stressing of the in situ acetabular component.

\section{Imageless navigation tool}

The IntelliJoint HIP navigation tool has been described in detail in previous studies. ${ }^{11}$ In short, the navigation tool consists of a miniature camera, a motion tracker and a computer workstation (Figure 1). The anterior pelvic plane is registered at the beginning of the operation using an alignment rod placed parallel to the patient's back. The camera is mounted on a pelvic platform that is in turn secured to the ipsilateral iliac crest using 2 threaded pins, creating a rigid couple such that any intraoperative movement of the pelvis will also move the camera. The camera captures the movement and position of the motion tracker, which can be magnetically fixed to the cup impactor to measure cup position, or to the greater trochanter to measure leg length and offset. The positions of in situ components are calculated by intraoperatively marking their borders using a navigated pointer device. Specifically, the navigation tool measures radiographic inclination and radiographic anteversion according to Murray's definitions. ${ }^{12}$ All data are then transferred to and displayed in real time on the laptop workstation.

\section{Radiographic analysis}

Two authors (X.Y.M., A.E.-R.) blinded to the intraoperative navigation measurements independently reviewed preoperative CT scans to measure the radiographic inclination (RI) and operative anteversion (OA) of the preexisting acetabular component according to Murray's definitions. RI was measured in the coronal plane as the angle between a line drawn parallel to the ischial tuberosities and a line connecting the most superior and the most inferior rims of the cup. OA was measured in the sagittal plane as the angle between the longitudinal axis of 


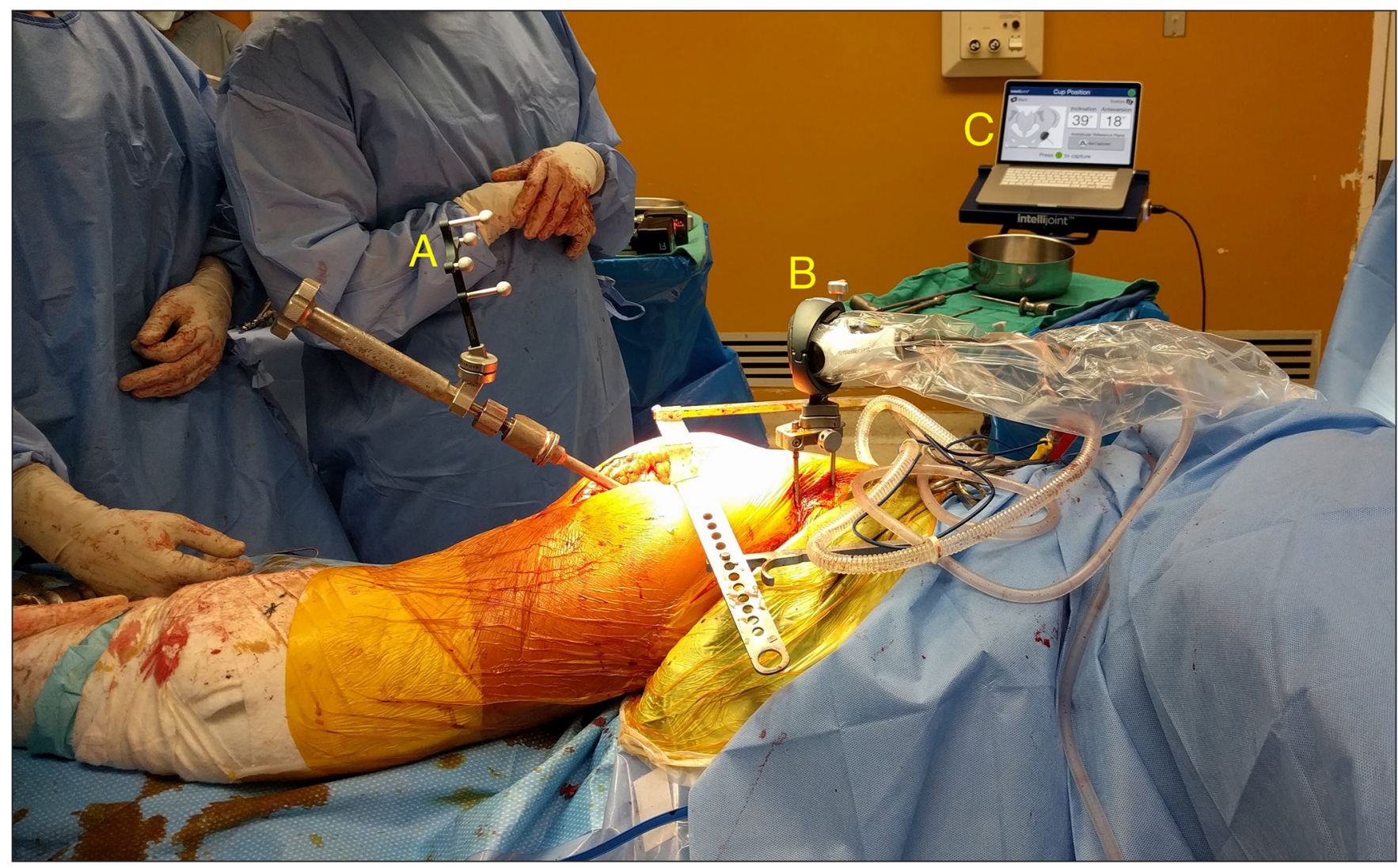

Fig. 1. Intraoperative photo of the navigation tool in use, demonstrating the positions of the mounted tracker (A), camera (B) and laptop workstation (C).

the patient's body and a line connecting the most superior and the most inferior rims of the cup. Radiographic anteversion (RA) was then calculated from the measured radiographic inclination and operative anteversion values using the conversion formula from Murray's original paper: $\tan (\mathrm{RA})$ equals $\cos (\mathrm{RI})$ multiplied by $\tan (\mathrm{OA}) .^{12}$ Final CT-measured inclination and anteversion were defined as the mean of the 2 authors' measurements.

\section{Statistical analysis}

Descriptive statistics were used to summarize the baseline demographic characteristics of the study cohort. The Pearson correlation coefficient with $95 \%$ confidence intervals (CIs) and paired $t$ test were used to assess the level of agreement between intraoperative and CT measurements of cup inclination and anteversion. Pearson correlation coefficients were characterized as poor (0.00-0.29), fair (0.30-0.59), moderate $(0.60-0.79)$ or very strong $(0.80-1.00) .{ }^{13}$ Bland-Altman plots were constructed to visualize the agreement between intraoperative and CT-measured values. Statistical analysis was performed using GraphPad Prism version 8.4.1 (GraphPad Software). A $p$ value of less than 0.05 was considered statistically significant.

\begin{tabular}{|lc|}
\begin{tabular}{|l|} 
Table 1. Surgical details \\
Element
\end{tabular} & $\begin{array}{c}\text { No. }(\%) \text { of patients } \\
n=53\end{array}$ \\
\hline Right side & $29(54.7)$ \\
\hline Approach & $36(67.9)$ \\
\hline Lateral & $3(5.7)$ \\
\hline Trochanteric slide & $14(26.4)$ \\
\hline Extended trochanteric osteotomy & \\
\hline Component revised & $20(37.7)$ \\
\hline Isolated acetabular & $12(22.6)$ \\
\hline Isolated femoral & $15(28.3)$ \\
\hline Head and liner exchange & $6(11.3)$ \\
\hline All components & \\
\hline Indication for revision & $20(37.7)$ \\
\hline Aseptic loosening & $7(13.2)$ \\
\hline Instability & $6(11.3)$ \\
\hline Poly wear & $4(7.5)$ \\
\hline Periprosthetic fracture & $4(7.5)$ \\
\hline Cup malposition & $3(5.7)$ \\
\hline Trunnionosis & $2(3.7)$ \\
\hline Trochanteric nonunion & $2(3.8)$ \\
\hline Leg length discrepancy & $1(1.9)$ \\
\hline Infection (first stage) & $1(1.9)$ \\
\hline Psoas tendinitis & $1(1.9)$ \\
\hline Painful hardware & $1(1.9)$ \\
\hline Loose liner & $1(1.9)$ \\
\hline Prior hip fusion & \\
\hline
\end{tabular}




\section{Results}

The study cohort consisted of 30 women and 23 men with a mean age of 66.3 years (standard deviation [SD] $12.4 \mathrm{yr}$, range 40 to $93 \mathrm{yr}$ ) and body mass index of $31.0 \mathrm{~kg} / \mathrm{m}^{2}$ (SD $6.6 \mathrm{~kg} / \mathrm{m}^{2}$, range 20 to $52 \mathrm{~kg} / \mathrm{m}^{2}$ ), respectively. The mean American Society of Anesthesiologists (ASA) classification at the time of surgery was 2.9 (SD 0.5 , range 2 to 4 ). A direct lateral approach to the hip was used in all patients (Table 1). Three patients $(5.7 \%)$ required a trochanteric slide osteotomy, and 14 patients $(26.4 \%)$ required an extended trochanteric osteotomy. The most common indication for revision was aseptic loosening $(n=20$, $37.7 \%)$. The most common procedure performed was isolated acetabular revision $(n=20,37.7 \%)$.

Excellent interrater reliability was observed between the 2 authors performing manual CT measurements for both cup inclination ( $r=0.96$ [95\% CI 0.93 to 0.98], $p<$ 0.001 ) and anteversion ( $r=0.94$ [95\% CI 0.89 to 0.96 ], $p<$ $0.001)$. The mean navigation-recorded inclination and anteversion were $42.1^{\circ}$ (SD $14.4^{\circ}$, range $-5^{\circ}$ to $71^{\circ}$ ) and $12.2^{\circ}$ (SD $17.2^{\circ}$, range $-54^{\circ}$ to $39^{\circ}$ ), respectively. The mean CT-measured inclination and anteversion were $41.7^{\circ}$ (SD $12.5^{\circ}$, range $-8.4^{\circ}$ to $70.5^{\circ}$ ) and $12.7^{\circ}$ (SD $19.5^{\circ}$, range $-68.1^{\circ}$ to $45.8^{\circ}$ ), respectively. Using CT measurements as the gold standard, only 15 of 53 (28.3\%) acetabular components had both inclination and anteversion angles within the Lewinnek safe zone. The mean absolute difference between navigation-recorded and CTmeasured component position was $5.2^{\circ}\left(\mathrm{SD} 4.0^{\circ}\right)$ for cup inclination and $4.8^{\circ}$ (SD 5.4 $)$ for cup anteversion. The intraoperative measurement was within $5^{\circ}$ of the radiographic measurement in 32 of $53(60.4 \%)$ hips for inclination and in 37 of $53(69.8 \%)$ hips for anteversion. The intraoperative measurement was within $10^{\circ}$ of the radiographic measurement in 47 of 53 (88.7\%) hips for inclination and 46 of $53(86.8 \%)$ hips for anteversion.

Very strong positive correlations were observed between navigation and CT measurements for both cup inclination $(r=0.89$ [95\% CI 0.81 to 0.93 ] $p<0.001)$ and anteversion $(r=0.93$ [95\% CI 0.88 to 0.96 ], $p<$ $0.001)$. Paired $t$ tests showed no significant difference between navigation and CT measurements for both inclination (mean of differences $0.41^{\circ}$ [SD 6.6 $6^{\circ}$, $p=$ 0.66 ) and anteversion (mean of differences $0.57^{\circ}$ SSD $\left.\left.7.2^{\circ}\right], p=0.57\right)$. Bland-Altman analysis demonstrated no significant proportional bias for both cup inclination (mean difference 0.40 [SD 6.6]; Figure 2) and anteversion (mean difference 0.57 [SD 7.2]; Figure 3).

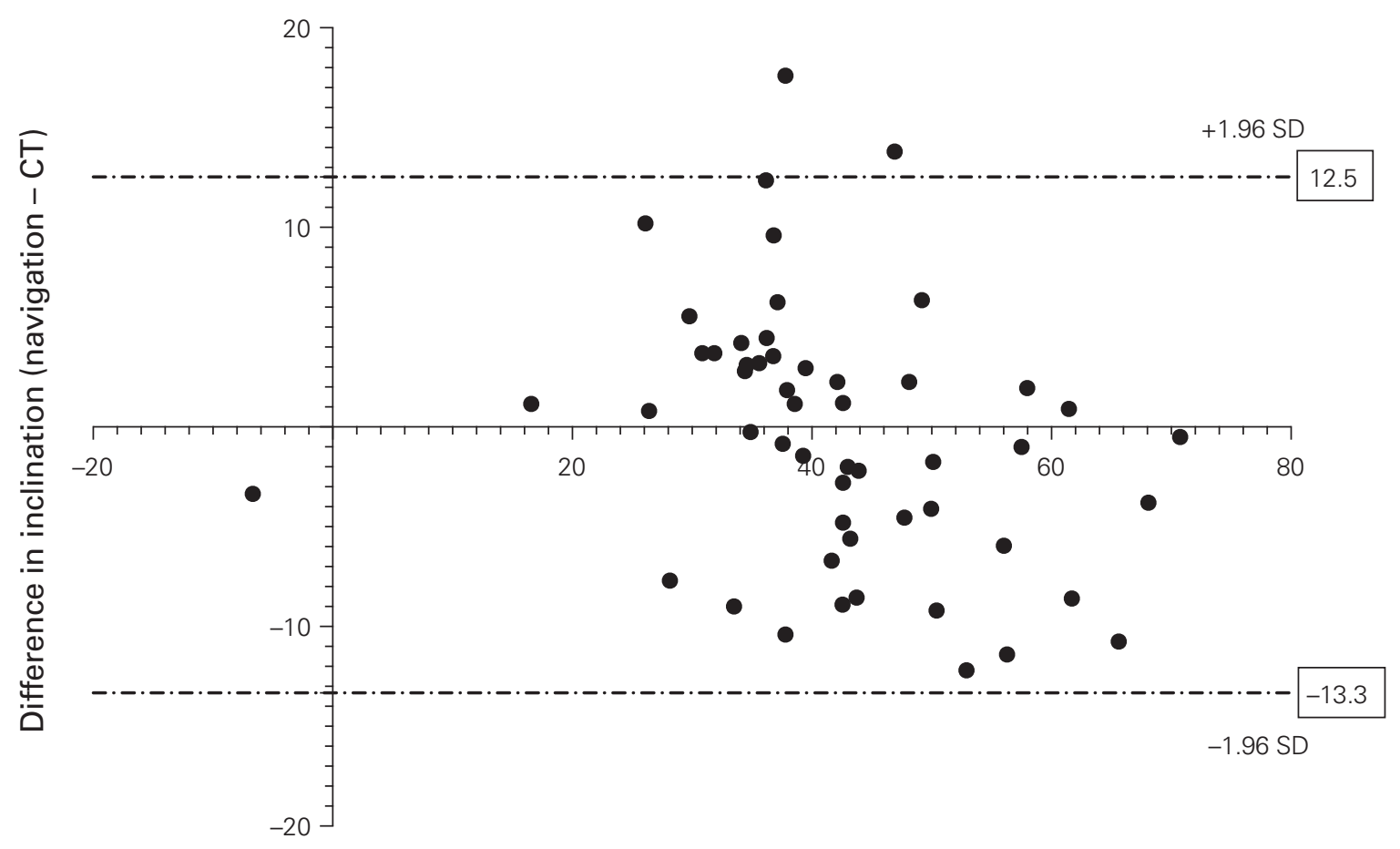

Mean inclination between navigation and CT measurements

Fig. 2. Bland-Altman plot showing the difference in cup inclination between navigation and CT measurements against the mean of the 2 measurements. CT = computed tomography; SD = standard deviation. 


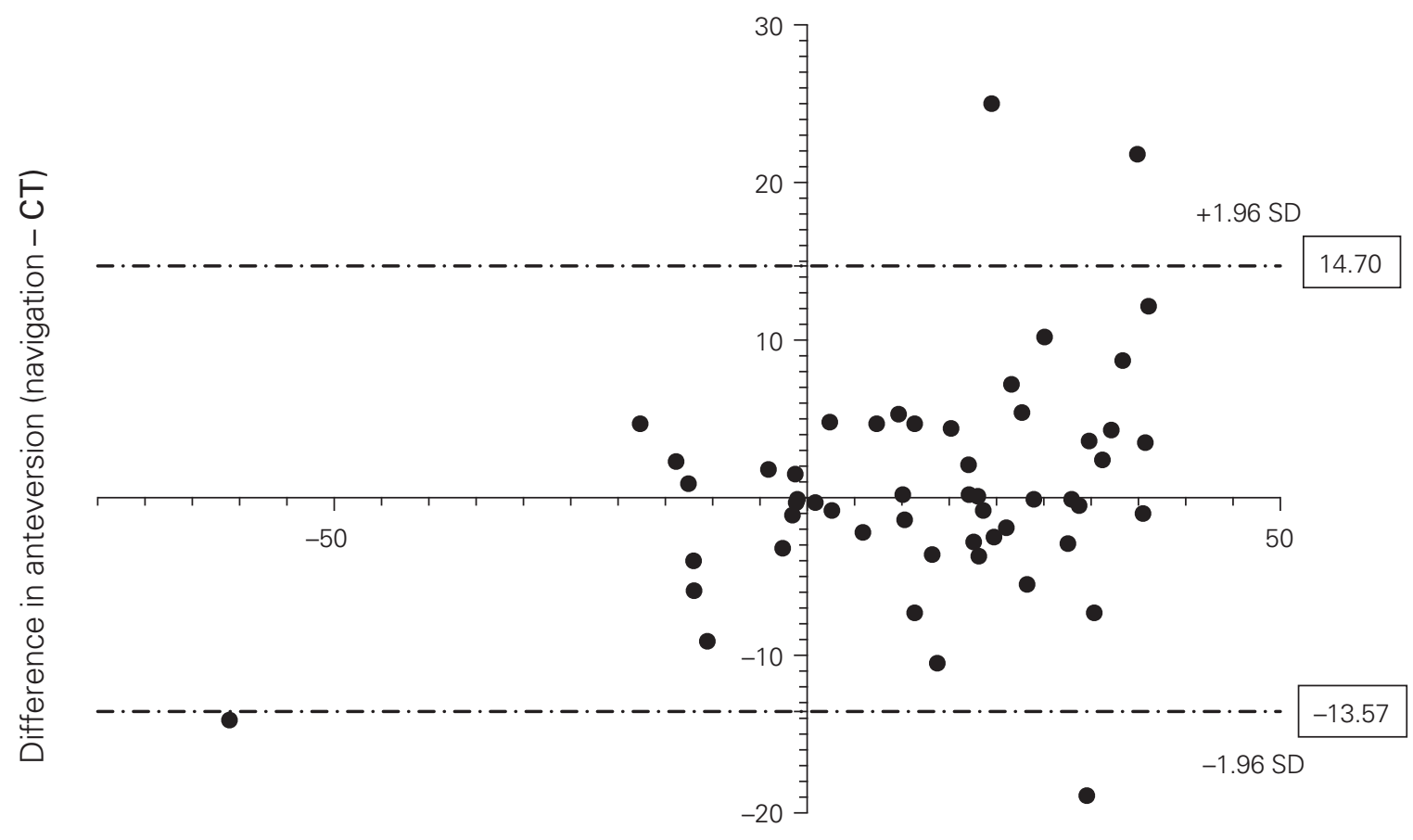

Mean anteversion between navigation and CT measurements

Fig. 3. Bland-Altman plot showing the difference in cup anteversion between navigation and CT measurements against the mean of the 2 measurements. $\mathrm{CT}=$ computed tomography; $\mathrm{SD}=$ standard deviation.

\section{Discussion}

Acetabular component malposition has been shown to increase the risk for postoperative dislocation, component impingement, accelerated bearing wear, and future revision surgery. ${ }^{14,15}$ Accurate cup placement is especially challenging in revision THA because of distorted anatomy, bone loss secondary to osteolysis and implant removal, as well as higher incidence of soft tissue scarring and contracture. Although many computer-assisted navigation tools have been shown to increase the accuracy of cup placement in primary THA, ${ }^{16,17}$ to our knowledge only 1 study to date has evaluated the accuracy of navigated cup placement during revision THA. ${ }^{18}$ We therefore sought to evaluate the accuracy of the imageless navigation tool Intellijoint HIP in measuring cup inclination and anteversion during revision THA, as compared with manual measurements using preoperative CT.

Our study found excellent agreement between navigation-recorded and manual CT measurements for both cup inclination and anteversion over a wide range of cup positions. Furthermore, there was no consistent underestimation or overestimation of cup inclination or anteversion by the navigation system. Our findings add to the existing literature on computer-assisted THA by demonstrating the accuracy of an imageless navigation tool in complex revision THA, as well as its ability to detect malposition of the preexisting acetabular component.

The large proportion of acetabular components found to be outside the Lewinnek safe zone is expected and reflects revision procedures involving aseptic loosening and possible component migration. The large number of patients requiring trochanteric osteotomy also reflects the technical complexity of revision THA. In the only published study to date on navigated revision THA, Chang and colleagues reported on 40 computer-assisted revision THAs using an imageless navigation system (VectorVision, BrainLab). Similar to our study, they found the mean prerevision cup inclination and anteversion to be $40.8^{\circ}$ (SD $14.2^{\circ}$, range $14.9^{\circ}$ to $81.4^{\circ}$ ) and $25.8^{\circ}$ (SD $15.4^{\circ}$, range $21.8^{\circ}$ to $55.3^{\circ}$ ), respectively, with 23 of $40(57.5 \%)$ cups outside their predetermined safe zone. ${ }^{18}$ These findings suggest that malposition of the preexisting acetabular component is common in revision THA and may present intraoperative decision-making challenges regarding revision versus retention of a well-fixed acetabular component. Extraction of well-fixed acetabular components with 
solid bony ingrowth is technically demanding and can increase blood loss and host bone loss, prolong operative time and increase the risk for infection. Navigation tools can facilitate intraoperative decision-making during revision THA as they not only allow identification of malpositioned components, but also help optimize the orientation of new revision components in cases requiring cup revision. This has been demonstrated in 2 case reports where data provided by the navigation tool were integral to intraoperative decision-making regarding cup revision versus retention. ${ }^{19,20}$

We observed a higher discrepancy between navigationrecorded and CT-measured values in our study $\left(5.2^{\circ}\right.$ [SD $4.0^{\circ}$ ] for inclination and $4.8^{\circ}$ [SD $5.4^{\circ}$ ] for anteversion) in comparison with previous studies involving navigated primary THA. Ybinger and colleagues reported on 37 navigated primary THAs and noted the mean difference between navigation and CT measurements to be $3.5^{\circ}\left(\mathrm{SD} 4.4^{\circ}\right.$ ) for inclination and $6.5^{\circ}$ (SD $7.3^{\circ}$ ) for anteversion. ${ }^{21}$ Similarly, Dorr and colleagues observed a mean difference of $4.4^{\circ}$ for inclination and $4.1^{\circ}$ for anteversion between navigation and CT measurements in a cohort of 30 navigated primary THAs. ${ }^{22}$ Possible explanations for the increased discrepancy between navigation and CT measurements seen in our study include the increased body mass index of our patient cohort (mean 31.0 [SD 6.6] $\mathrm{kg} / \mathrm{m}^{2}$; range 20 to $52 \mathrm{~kg} / \mathrm{m}^{2}$ ), as well as the distorted anatomy associated with complex revision procedures. Imageless navigation relies on accurate registration of bony landmarks to calculate the anterior pelvic plane for use as a reference for acetabular component position..$^{23}$ Patients undergoing revision THA often present with distorted anatomy such as substantial pelvic obliquity and pelvic tilt, soft tissue contractures and periprosthetic osteolysis or fractures that can interfere with accurate registration of bony landmarks and lead to intraoperative measurement errors. Furthermore, obesity has been shown to be associated with decreased accuracy of navigated cup placement, probably because of the obscuring of bony landmarks by overlying soft tissue, resulting in measurement error. ${ }^{17,21}$

\section{Limitations}

This study has several limitations. First, we measured and compared the position of the preexisting acetabular component rather than that of the new revision component in cases involving cup revision. Although this eliminated the risk of patient morbidity secondary to navigation error and allowed for testing of navigation accuracy at extreme cup positions outside the Lewinnek safe zone, it limited the ability to extrapolate our results to the insertion of new revision cups. Furthermore, although intraoperative measurements were taken before extensive scar tissue débridement and manual stressing of the preexisting acetabular component, there remained the possibility of cup migration between preoperative CT and intraoperative measurement in cases involving acetabular osteolysis and aseptic loosening, which may have confounded our results. Thus, further studies evaluating the position of newly inserted revision cups are warranted. Second, the maximum clinically acceptable underestimation or overestimation of cup inclination and anteversion by navigation tools in THA remains poorly defined. Although 2 small studies have used $5^{\circ}$ as the maximum clinically acceptable margin of error for navigation in hip resurfacing, ${ }^{24,25}$ no such value has been proposed for THA. Given the distorted anatomy and bone loss routinely encountered in revision THA and the availability of specialized components such as dual mobility cups and constrained liners, we accepted a $10^{\circ}$ margin of error. However, we acknowledge that the clinically acceptable margin of error may vary among surgeons, and thus this limits the strength of our conclusions. Finally, we did not report on clinical outcomes but confined our study to radiographic findings.

\section{Conclusion}

Imageless navigation demonstrated excellent correlation and agreement with CT measurements for both cup inclination and anteversion over a wide range of acetabular component positions and patient body habitus. However, its accuracy in revision THA may be lower than its accuracy in primary THA. Further study is needed to elucidate the limitations of and specific contraindications for imageless navigation during revision THA and to evaluate whether navigation results in a reduction of adverse events and improved clinical outcomes.

Affiliations: From the Division of Orthopaedic Surgery, Mount Sinai Hospital, Toronto, Ont.

Competing interests: A. Gross holds stock and stock options in Intellijoint Surgical. No other competing interests were declared.

Contributors: X. Mei, O. Safir, A. Gross and P. Kuzyk designed the study. X. Mei and A. Etemad-Rezaie acquired the data, which X. Mei, A. Etemad-Rezaie and P. Kuzyk analyzed. X. Mei and A. Etemad-Rezaie wrote the article, which all authors critically revised. All authors approved the final version to be published.

Content licence: This is an Open Access article distributed in accordance with the terms of the Creative Commons Attribution (CC BY-NC-ND 4.0) licence, which permits use, distribution and reproduction in any medium, provided that the original publication is properly cited, the use is noncommercial (i.e., research or educational use), and no modifications or adaptations are made. See: https://creativecommons.org/licenses/by-nc-nd/4.0/

\section{References}

1. Bozic KJ, Kurtz SM, Lau E, et al. The epidemiology of revision total hip arthroplasty in the United States. 7 Bone foint Surg Am 2009;91:128-33. 
2. Dorr LD, Callaghan JJ. Death of the Lewinnek "safe zone." 7 Arthroplasty 2019;34:1-2.

3. Lewinnek GE, Lewis JL, Tarr R, et al. Dislocations after total hipreplacement arthroplasties. F Bone foint Surg Am 1978;60:217-20.

4. Digioia AM III, Jaramaz B, Plakseychuk AY, et al. Comparison of a mechanical acetabular alignment guide with computer placement of the socket. F Arthroplasty 2002;17:359-64.

5. Hassan DM, Johnston GH, Dust WN, et al. Accuracy of intraoperative assessment of acetabular prosthesis placement. $\mathcal{f}$ Arthroplasty 1998;13:80-4.

6. DiGioia AM, Jaramaz B, Blackwell M, et al. The Otto Aufranc Award. Image guided navigation system to measure intraoperatively acetabular implant alignment. Clin Orthop Relat Res 1998;355:8-22.

7. Leenders T, Vandevelde D, Mahieu G, et al. Reduction in variability of acetabular cup abduction using computer assisted surgery: a prospective and randomized study. Comput Aided Surg 2002;7:99-106.

8. Sugano N, Nishii T, Miki H, et al. Mid-term results of cementless total hip replacement using a ceramic-on-ceramic bearing with and without computer navigation. 7 Bone foint Surg Br 2007;89:455-60.

9. Bradley MP, Benson JR, Muir JM. Accuracy of acetabular component positioning using computer-assisted navigation in direct anterior total hip arthroplasty. Cureus 2019;11:e4478.

10. Christ A, Ponzio D, Pitta M, et al. Minimal increase in total hip arthroplasty surgical procedural time with the use of a novel surgical navigation tool. Open Orthop 7 2018;12:389-95.

11. Paprosky WG, Muir JM. Intellijoint HIP ${ }^{\circledR}$ : a 3D mini-optical navigation tool for improving intraoperative accuracy during total hip arthroplasty. Med Devices (Auckl) 2016;9:401-8.

12. Murray DW. The definition and measurement of acetabular orientation. $\mathcal{F}$ Bone foint Surg Br 1993;75:228-32.

13. Akoglu H. User's guide to correlation coefficients. Turk 7 Emerg Med 2018;18:91-93.

14. Kennedy JG, Rogers WB, Soffe KE, et al. Effect of acetabular component orientation on recurrent dislocation, pelvic osteolysis, polyethylene wear, and component migration. I Arthroplasty 1998;13:530-4.
15. Moskal JT, Capps SG. Improving the accuracy of acetabular component orientation: avoiding malposition. 7 Am Acad Orthop Surg 2010;18:286-96.

16. Haaker RG, Tiedjen K, Ottersbach A, et al. Comparison of conventional versus computer-navigated acetabular component insertion. $\mathcal{F}$ Arthroplasty 2007;22:151-9.

17. Parratte S, Argenson JN. Validation and usefulness of a computerassisted cup-positioning system in total hip arthroplasty. A prospective, randomized, controlled study. 7 Bone foint Surg Am 2007;89:494-9.

18. Chang JD, Kim IS, Prabhakar S, et al. Revision total hip arthroplasty using imageless navigation with the concept of combined anteversion. F Arthroplasty 2017;32:1576-80.

19. Paprosky WG, Vincent J, Sostak JR, et al. Computer-assisted navigation as a diagnostic tool in revision total hip arthroplasty: a case report. SAGE Open Med Case Rep 20197:2050313X19827743.

20. Vincent J, Alshaygy I, Muir JM, et al. Preservation of the acetabular cup during revision total hip arthroplasty using a novel mininavigation tool: a case report. 7 Orthop Case Rep 2018;8:53-56.

21. Ybinger T, Kumpan W, Hoffart HE, et al. Accuracy of navigationassisted acetabular component positioning studied by computed tomography measurements: methods and results. 7 Arthroplasty 2007;22:812-7.

22. Dorr LD, Malik A, Wan Z, et al. Precision and bias of imageless computer navigation and surgeon estimates for acetabular component position. Clin Orthop Relat Res 2007;465:92-9.

23. Lass R, Kubista B, Olischar B, et al. Total hip arthroplasty using imageless computer-assisted hip navigation: a prospective randomized study. F Artbroplasty 2014;29:786-91.

24. Weenders SG, van de Kimmenade RJ, Gratama JW, et al. Hip resurfacing implant orientation analysis: a comparison of a computer-added design technique and computed tomography. $f$ Arthroplasty 2016;31:501-5.

25. Westacott DJ, McArthur J, King RJ, et al. Assessment of cup orientation in hip resurfacing: a comparison of TraumaCad and computed tomography. 7 Orthop Surg Res 2013;8:8. 